\title{
Refractory celiac disease type 2: how to diagnose and treat?
}

\author{
Jonathan Soldera1* (1), Karina Salgado² (D), Karla Lais Pêgas ${ }^{2,3}$
}

\section{SUMMARY}

Refractory celiac disease is an uncommon condition which might be associated to poor prognosis. It is often treated with immunosuppressive medications, with poor results. It is divided in type 1 and type 2, the latter carrying a high risk for lymphoma and mortality. A case of a 41 year old female patient with refractory celiac disease type 2 is reported. She was treated with oral budesonide for six months, achieving histological remission.

KEYWORDS: Celiac disease. Budesonide. Diet, gluten-free.

\section{INTRODUCTION}

Celiac disease (CD) is defined as an immune-mediated disease, which affects genetically predisposed individuals. The immune response is triggered by the ingestion of gluten, which is present in cereals such as wheat, rye, and barley. In countries with high proportion of European descendants, it is estimated to affect $1 \%$ of the population as its incidence and prevalence has been rising $^{1-3}$. Diagnosis of CD is made by an association of positive antibodies (generally, anti-transglutaminase for adults and anti-gliadin for children) and a duodenal biopsy demonstrating villous atrophy and an increase in intraepithelial lymphocytes (IELs) amount. The only treatment currently available is lifelong gluten-free diet (GFD), which normalizes antibodies and duodenal histology and improves symptoms ${ }^{1-3}$. Steroids are generally reserved at presentation only for celiac crisis ${ }^{4}$.

Refractory celiac disease (RCD) is a severe and uncommon presentation of CD, occurring in $<2 \%$ of celiac patients. It should be suspected in a patient who still has symptoms of malabsorption and villous atrophy on duodenal biopsies, even after at least 6 months of GFD ${ }^{1}$. It is paramount to confirm that GFD orientation is being strictly followed and to exclude malignancy and other causes of nonresponse to GFD.

The suspicion of RCD should prompt immunohistochemistry and/or flow cytometry of the duodenal biopsies, in order to stratify RCD into two types (Table 1 ). RCD type 1 is associated with normal CD3, CD4, and CD8 surface markers on lymphocytes, without clonal gene rearrangement of the gamma chain of the T-cell receptor (TCR). RCD type 2 is associated with aberrant clonal IELs that lack surface expression of CD3, CD4, and CD8 and is associated with TCR gamma gene rearrangement ${ }^{2}$. RCD type 2 carries a higher risk for T-cell lymphoma and mortality, and it is treated with strict GFD and the use of immunosuppressive medications, although with poor results ${ }^{3}$.

The purpose of this study is to report a case of a female patient diagnosed with CD in an uncommon situation, during an investigation of chronic alteration of serum amylases. She was later diagnosed with RCD type 2 after 2 years of follow-up and went into remission with the use of oral budesonide, a novel treatment option for these cases.

\footnotetext{
'Universidade de Caxias do Sul - Caxias do Sul (RS), Brazil.

¿2Universidade Federal de Ciências da Saúde de Porto Alegre - Porto Alegre (RS), Brazil.

${ }^{3}$ Irmandade Santa Casa de Misericórdia de Porto Alegre - Porto Alegre (RS), Brazil.

*Corresponding author: jonathansoldera@gmail.com

Conflicts of interest: the authors declare there are no conflicts of interest. Funding: none.

Received on October 15, 2020. Accepted on November 16, 2020.
} 


\section{DESCRIPTION}

A female patient, 41 years old, previously healthy, sought care due to unspecific abdominal pain, weight loss, and elevation of pancreatic enzymes for more than 1 year. Workup had shown normal abdominal magnetic resonance imaging (MRI) and computerized tomography, serum amylase level at $276 \mathrm{U} / \mathrm{L}$ (reference $=120 \mathrm{U} / \mathrm{L}$ ), lipase values of $16 \mathrm{U} / \mathrm{L}$, and a negative in parasitological stool examination test. Anti-transglutaminase IgA was $128 \mathrm{U} / \mathrm{mL}$ (reference $=10 \mathrm{U} / \mathrm{mL}$ ), and anti-gliadin $\operatorname{IgA}$ was $212 \mathrm{U} / \mathrm{mL}$ (reference $=30 \mathrm{U} / \mathrm{mL}$ ). She underwent upper digestive endoscopy, which showed duodenum with severe atrophy, with MARSH IIIb in biopsies. The patient was oriented to start following GFD and showed some improvement of symptoms and normalization of serum amylases.

After 2 years of strict GFD with a follow-up by an experienced dietitian, she still presented daily mild abdominal pain, anemia, and occasional watery diarrhea, with normalization of anti-transglutaminase IgA and anti-gliadin IgA. Abdominal MRI was normal. Upper digestive endoscopy showed atrophic duodenum with MARH IIIa in biopsies (Figure 1A). Immunohistochemistry was performed, showing positive $\mathrm{CD} 3$ in the IELs and positive CD8 in 50\% or less of IELs stained by CD3 (Figure 2). She was then diagnosed with RCD type 2 . Her adherence to GFD was reviewed and confirmed again by another dietitian, and oral budesonide (OB) in the dose of $3 \mathrm{mg}$ three times a day $\left(\right.$ Entocort $\left.^{\circledR}\right)$ was started, as stated in the study by Mukewar et al.: "Directions for taking OB were as follows:

1. first daily capsule: open the capsule, empty contents into applesauce and stir, grind the medicine between the teeth, rinse and swallow with a glass of water;

2. second daily capsule: open the capsule, empty contents into applesauce, stir, rinse and swallow with a glass of water;

3. third daily capsule: swallow the whole capsule"1.

After 6 months of treatment with $\mathrm{OB}$, upper digestive endoscopy showed recovery of duodenal atrophy (Figure 1B), with MARSH I on biopsies (Figure 3). She was successfully tapered off OB and remained asymptomatic on strict GFD after 2 years of follow-up.

\section{DISCUSSION}

In this case, summarized in Table 2, two important topics of CD are discussed: uncommon presentations, such as an unspecific

Table 1. Diagnostic findings for refractory celiac disease type 1 and 2 on duodenal biopsy immunochemistry or flow cytometry

\begin{tabular}{|c|c|c|}
\hline $\mathrm{RCD}$ & Type 1 & Type 2 \\
\hline IEL phenotype & $\begin{array}{c}\text { IEL are } C D 3+/ C D 8+\text {. } \\
\text { CD3+/CD8 }-<40-50 \% \text { by } \\
\text { immunohistochemistry, and }<20-25 \% \\
\text { by flow cytometry. }\end{array}$ & $\begin{array}{c}\text { IEL mostly aberrant. } \\
\text { IEL CD3+/CD8- }>40-50 \% \text { by } \\
\text { immunohistochemistry, and }>20-25 \% \\
\text { by flow cytometry. }\end{array}$ \\
\hline $\begin{array}{l}\text { TCR Gamma gene rearrangement } \\
\text { polymerase chain reaction }\end{array}$ & Polyclonal TCR & Monoclonal TCR \\
\hline
\end{tabular}

RCD: refractory celiac disease; IEL: intra-epithelial lymphocyte; TCR: T-cell receptor.

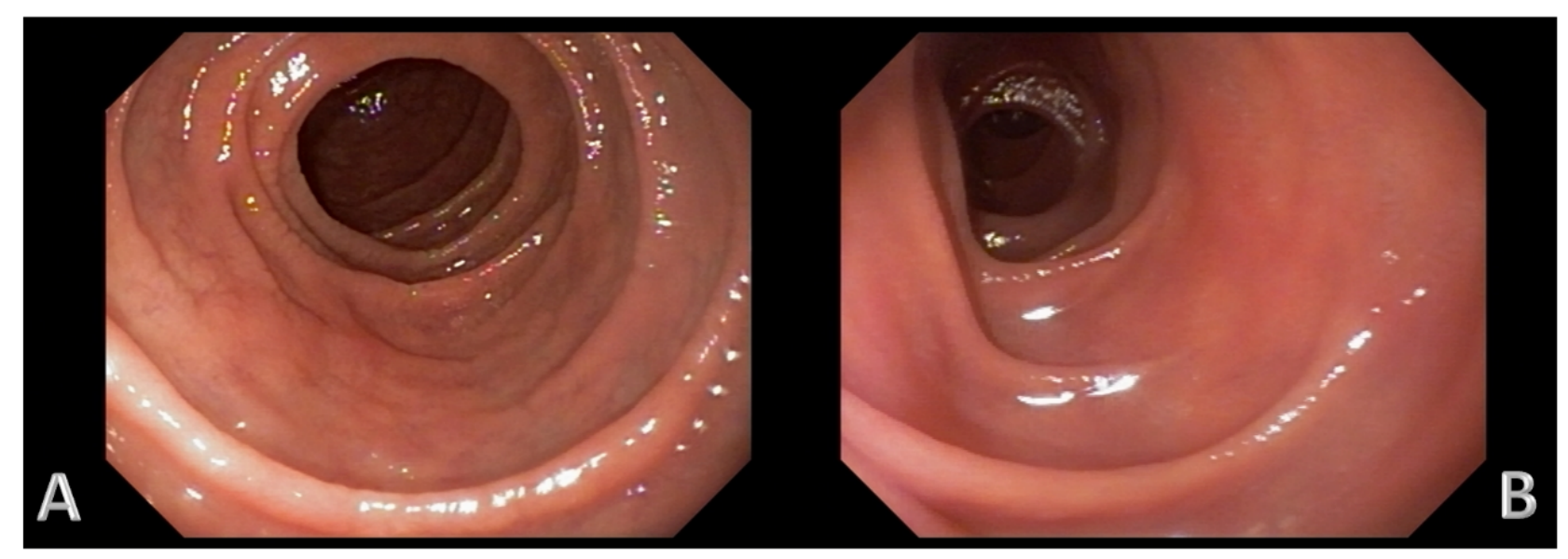

Figure 1. Upper digestive endoscopy (duodenum). (A) Duodenal atrophy and severe scalloping of duodenal folds; (B) Resolution of duodenal lesion. 
abdominal pain associated with unspecific laboratory alterations, and RCD, which prevalence is bound to increase as $\mathrm{CD}$ prevalence continues to grow worldwide.

Most patients diagnosed with CD improve greatly with GFD, and complete clinical and histological response does not occur in every celiac patient. A small group of these patients will present persistent duodenal atrophy and recurrent symptoms (such as, anemia, diarrhea, abdominal pain, and weight loss), despite strict adherence to GFD ${ }^{1,2}$. RCD is defined by persistent or recurrent symptoms associated with villous atrophy and increased IELs despite 6-12 months of strict confirmed GFD, after the exclusion of malignancy and other causes of lack or loss of response to GFD ${ }^{1,2}$. Causes that should be considered in this step of differential diagnosis are: tropical sprue, autoimmune enteropathy, hypogammaglobulinemia, idiopathic AIDS enteropathy, eosinophilic gastroenteritis, Whipple disease, intestinal T-cell lymphoma, ulcerative jejunitis, collagenous sprue, giardiasis, other parasitic infections, and ischemic enteritis ${ }^{2,4,5}$.

$\mathrm{RCD}$ is considered an uncommon condition, which might be related to a poor prognosis. In order to better stratify prognosis, it is divided into type 1 and type $2^{2,3,6}$. It is sub-classified by the detection of abnormal IEL phenotype, which helps clarify prognosis. The current methods can be done in fixed (double CD3/CD8 immunohistochemistry and TCR clonal rearrangement by $\mathrm{PCR}$ ) or on fresh frozen intestinal tissue (flow cytometry). The abnormal phenotype is supported by the loss of normal surface markers CD3, CD4, and CD8 with preserved expression of intracytoplasmic CD3 in more than $50 \%$ of IELs as evaluated by immunohistochemistry ${ }^{1}$ or more than $20-25 \%$ as determined by flow cytometry and detection of TCR chains clonal rearrangement by PCR ${ }^{2,6}$.

Abnormal IELs are the hallmark of RCD type 2, which is associated with poor prognosis because of a higher risk of progression to T-cell lymphoma ${ }^{1-3,5,6}$. This was the major limitation of the reported case: due to the unavailability of flow cytometry, diagnosis of RCD type 2 was made using immunohistochemistry, which is a method that can reveal dominant aberrant

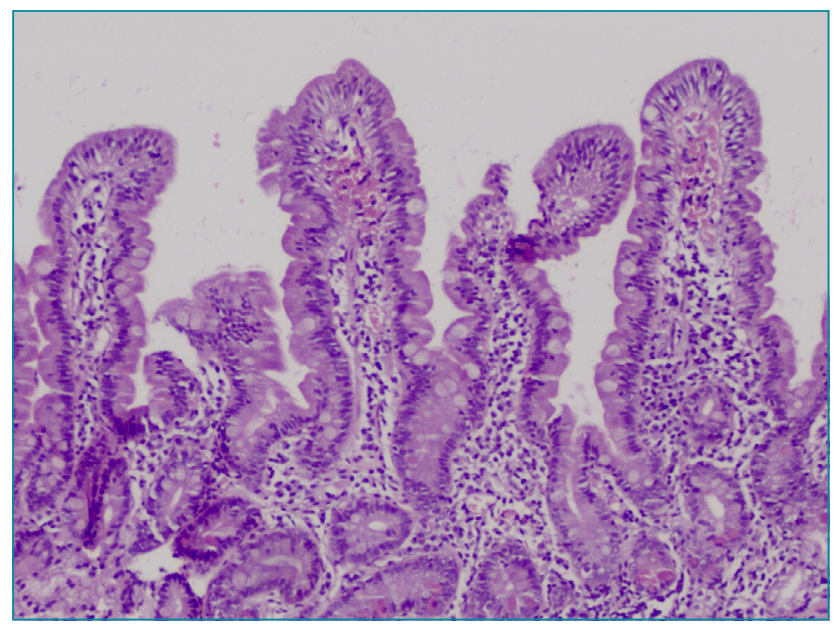

Figure 3. Duodenal biopsy (H.E, 100x). Recovery of the villous architecture, with discrete increase in IEL infiltrates (MARSH I).

Table 2. Summary of the reported case.

\begin{tabular}{l|c}
\hline $\begin{array}{l}\text { Clinical } \\
\text { presentation }\end{array}$ & $\begin{array}{c}41 \text { years old female patient. } \\
\text { Abdominal pain. } \\
\text { Elevation of serum amylases. }\end{array}$ \\
\hline $\begin{array}{l}\text { Diagnostic } \\
\text { tests }\end{array}$ & $\begin{array}{c}\text { Normal computed tomography. } \\
\text { Celiac disease serology positive. } \\
\text { Duodenal biopsy: MARSH Illb. }\end{array}$ \\
\hline Evolution & $\begin{array}{c}\text { Persistent abdominal pain. } \\
\text { Strict adherence to gluten-free diet. } \\
\text { After 2 years of diagnosis: MARSH IIla. }\end{array}$ \\
\hline Final & $\begin{array}{c}\text { Immunohistochemistry: RCD type 2. } \\
\text { treatment } \\
\text { MARSH I at the end of treatment. }\end{array}$ \\
\hline
\end{tabular}

RCD: refractory celiac disease.

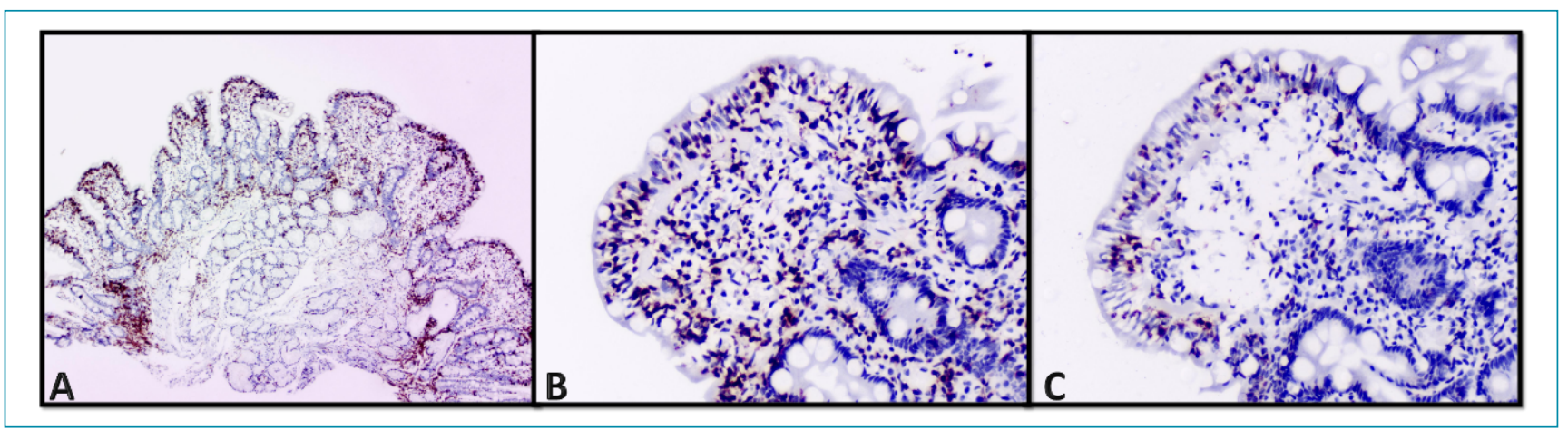

Figure 2. Duodenal biopsy and immunohistochemistry. (A) (anti-CD3 T lymphocytes, 100x): Villous with partial atrophy, fusion, and high number of IELs; (B) (anti-CD3 T Lymphocytes, 200x): Intraepithelial lymphocytosis (more than 30 lymphocytes/100 enterocytes); (C) (anti-CD8 T lymphocytes, 200x): Intraepithelial lymphocytosis in duodenal biopsy. The number of lymphocytes highlighted by antibody anti-CD8 is significantly lower than those stained with anti-CD3 (Figure 2A). 
IEL populations only when they make up over $50 \%$ of IELs ${ }^{7}$. However, in patients with a moderate aberrant IEL population (between 20 and 50\%), a significant number of patients will be undetected $^{8,9}$. The lack of sensitivity of immunohistochemistry can be largely explained by the inability of this method to distinguish between cell surface and intracellular expression of CD3, the latter being the hallmark of aberrant IELs, whereas flow cytometric analysis can differentiate cytoplasmic from membranous $\mathrm{CD} 3$ expression. Therefore, it is a superior method to identify patients at a higher risk for T-cell lymphoma, since it can better analyze TCR clonality 5 . Immunohistochemistry also has two major problems: it lacks specificity and an underlying disease can be inadequately classified as RCD type $2^{10}$; and it has a higher inter-observer variability ${ }^{11}$. This lower sensitivity should be outweighed against the fact that this technique is easily applicable and readily available, whereas flow cytometric analysis of IELs requires fresh duodenal biopsies and skilled analysts ${ }^{11}$.

$\mathrm{CD}$ is considered to be an uncommon cause of nonpancreatic elevation of amylases and lipases-this laboratorial finding is believed to occur in about $25 \%$ of celiac patients ${ }^{12}$. In a study comprised of 54 celiac patients, most of the elevated values were lower than twofold the reference values. After 12 months of GFD, serum amylase level was elevated in three cases and lipases in two cases, and these patients had not strictly adhered to the $\mathrm{GFD}^{12}$. It is reasonable, therefore, to screen patients with elevated serum amylases or lipases levels for $\mathrm{CD}$, in the absence of signs of pancreatic disease.

Although GFD is being strictly followed by CD patients, mortality when compared to non-celiac controls is still higher, with a hazard ratio of 1.21. This higher risk of death is associated with cardiovascular disease, cancer, and respiratory disease $\mathrm{e}^{13}$. This mortality increases even more in the presence of RCD. The 5-year survival rates described vary from $80 \%$ to $96 \%$ for RCD type 1 and $44 \%$ to $58 \%$ for RCD type $2^{14}$. Evolution to T-cell lymphoma in RCD type 2 generally occurs in patients with advanced age and if a late diagnosis of $\mathrm{CD}$ has been made. In this context, increased levels of lactate dehydrogenase and $\beta$-2-microglobulin might suggest that RCD has evolved to lymphoma, a life-threating complication of RCD type $2^{15}$.

RCD is often treated with immunosuppressive medications. Prednisone $(0.5-1 \mathrm{mg} / \mathrm{kg} / \mathrm{day})$ or a combination of prednisone and azathioprine $(2 \mathrm{mg} / \mathrm{kg} /$ day $)$ are clinically effective for most patients with RCD type 1 . Clinical response to steroids is observed in the majority of patients with RCD type 2. However, mucosal recovery is infrequent, and progression to T-cell lymphoma might not be totally preventable ${ }^{1,2,5,6}$. Therefore, current immunosuppressive treatments for RCD type 2, such as azathioprine, achieve poor results and might increase the risk of T-cell lymphoma.

In a study developed at Mayo Clinic by Mukewar et al., 43 patients with RCD type 1 and 13 patients with RCD type 2 were enrolled. There were two deaths related to lymphoma: one patient with RCD type 1 and one with RCD type 2 . All patients were treated with OB, with a response of $92 \%$ in both RCD type 1 and $2^{1}$. It is expected a recovery of duodenal mucosa with treatment with $\mathrm{OB}$ in a 6-month interval, although some patients might not be tapered off the medication ${ }^{1}$.

In another study, published by Therrien et al., 42 patients with non-responsive $\mathrm{CD}$ treated with budesonide $9 \mathrm{mg}$ daily were showed an inferior result compared with the study by Mukevar et al. It was found that, within 1 year of therapy, $25 \%$ of patients had clinical and mucosal recovery and $17 \%$ had both persistent symptoms and mucosal damage ${ }^{16}$.

\section{CONCLUSION}

In conclusion, it is significant to screen celiac patients with persistent symptoms or villous atrophy for RCD. This screening should accompany an extensive review of the adherence to GFD. Afterwards, with the use of immunohistochemistry and/or flow cytometry, these patients should be stratified into RCD type 1 or type 2 , with an objective of prognosticating the disease. If RCD type 2 is present, the physician should be aware of the higher risk of T-cell lymphoma. OB should then be considered as an effective treatment with a low rate of adverse effects.

\section{AUTHORS" CONTRIBUTIONS}

JS: Conceptualization, Writing - Original Draft, Writing Review \& Editing. KS: Data Curation, Writing - Original Draft, Writing - Review \& Editing. KLP: Data Curation, Writing - Original Draft, Writing - Review \& Editing.

\section{REFERENCES}

1. MukewarSS, Sharma A, Rubio-TapiaA, Wu TT, Jabri B, Murray JA. Opencapsule budesonide for refractory celiac disease. Am J Gastroenterol. 2017;112(6):959-67. https://doi.org/10.1038/ajg.2017.71
2. Rubio-Tapia A, Murray JA. Classification and management of refractory coeliac disease. Gut. 2010;59(4):547-57. https://doi. org/10.1136/gut.2009.195131 
3. West J. Celiac disease and its complications: a time traveller's perspective. Gastroenterology. 2009;136(1):32-4. https://doi. org/10.1053/j.gastro.2008.11.026

4. Soldera J, Coelho GP, Heinrich CF. Life-threatening diarrhea in an elderly patient. Gastroenterology. 2020;7:S00165085(20)35026-5. https://doi.org/10.1053/j.gastro.2020.07.054

5. Al-Toma A, Volta U, Auricchio R, Castillejo G, Sanders DS, Cellier $C$, et. al. European Society for the Study of Coeliac Disease (ESsCD) guideline for coeliac disease and other gluten-related disorders. United European Gastroenterol J. 2019;7(5):583613. https://doi.org/10.1177/2050640619844125

6. Malamut $G$, Afchain P, Verkarre V, Lecomte $T$, Amiot A, Damotte $D$, et al. Presentation and long-term follow-up of refractory celiac disease: comparison of type I with type II. Gastroenterology. 2009;136(1):81-90. https://doi.org/10.1053/j. gastro.2008.09.069

7. Serre NPM, Cellier C, Jabri B, Delabesse E, Verkarre V, Roche $B$, et al. Distinction between coeliac disease and refractory sprue: a simple immunohistochemical method. Histopathology. 2000;37(1):70-7. https://doi.org/10.1046/ j.1365-2559.2000.00926.x

8. van Wanrooij RL, Schreurs MW, Bouma G, von Blomberg BM, Tack GJ, Verbeek WH, et al. Accurate classification of RCD requires flow cytometry. Gut. 2010;59(12):1732. https://doi. org/10.1136/gut.2010.223438

9. Liu H, Brais R, Lavergne-Slove A, Jeng Q, Payne $K$, Ye H, et al. Continual monitoring of intraepithelial lymphocyte immunophenotype and clonality is more important than snapshot analysis in the surveillance of refractory coeliac disease. Gut. 2010;59(4):452-60. https://doi.org/10.1136/ gut.2009.186007
10. Malamut G, Meresse B, Cellier C, Cerf-Bensussan N Refractory celiac disease: from bench to bedside. Semin Immunopathol. 2012;34(4):601-13. https://doi.org/10.1007/ s00281-012-0322-z

11. van Wanrooij RL, Müller DM, Neefjes-Borst EA, Meijer J, Koudstaal LG, Heideman DA, et al. Optimal strategies to identify aberrant intra-epithelial lymphocytes in refractory coeliac disease. J Clin Immunol. 2014;34(7):828-35. https:// doi.org/10.1007/s10875-014-0075-7

12. Carroccio $A$, Di Prima $L$, Scalici $C$, Soresi $M$, Cefalù $A B$, Noto $D$, et al. Unexplained elevated serum pancreatic enzymes: a reason to suspect celiac disease. Clin Gastroenterol Hepatol. 2006;4(4):455-9. https://doi.org/10.1016/j.cgh.2005.12.027

13. Lebwohl B, Green PHR, Söderling J, Roelstraete B, Ludvigsson JF. Association between celiac disease and mortality risk in a swedish population. JAMA. 2020;323(13):1277-85. https:// doi.org/10.1001/jama.2020.1943

14. Nijeboer P, van Wanrooij R, van Gils T, Wierdsma NJ, Tack GJ, Witte $\mathrm{Bl}$, et al. Lymphoma development and survival in refractory coeliac disease type II: histological response as prognostic factor. United European Gastroenterol J. 2017;5(2):208-17. https://doi.org/10.1177/2050640616646529

15. Volta U, Caio G, De Giorgio R. Mistakes in refractory coeliac disease and how to avoid them. Vienna: UEG Education; 2019. [cited on Oct. 15, 2020]. Available from: https:// ueg.eu/a/149

16. Therrien A, Silvester JA, Leonard MM, Leffler DA, Fasano A, Kelly CP. Enteric-release budesonide may be useful in the management of non-responsive celiac disease. Dig Dis Sci. 2021;66(6):1989-97. https://doi.org/10.1007/s10620020-06454-5 